\title{
Letter
}

\section{Evaluation of handwriting peculiarities utilizing laser speckle contrast imaging}

\author{
Yuri Kuznetsov ${ }^{1}$, Anton Sdobnov ${ }^{2}$, Igor Meglinski ${ }^{2,3,4,5,6}$, Alon Harmelin ${ }^{1}$ \\ and Vyacheslav Kalchenko ${ }^{1,7}$ \\ 1 Department of Veterinary Resources, Weizmann Institute of Science, Rehovot, 76100, Israel \\ 2 Optoelectronics and Measurement Techniques Laboratory, University of Oulu, Oulu, 90570, Finland \\ 3 Interdisciplinary Laboratory of Biophotonics, National Research Tomsk State University, Tomsk \\ 634050, Russia \\ ${ }^{4}$ Institute of Engineering Physics for Biomedicine (PhysBio), National Research Nuclear University \\ (MEPhI), Moscow, 115409, Russia \\ 5 School of Engineering and Applied Science, Aston Institute of Materials Research, Aston University, \\ Birmingham, B4 7ET, United Kingdom \\ ${ }^{6}$ School of Life and Health Sciences, Aston University, Birmingham, B4 7ET, United Kingdom \\ E-mail: a.kalchenko@weizmann.ac.il
}

Received 19 July 2019

Accepted for publication 10 September 2019

Published 2 October 2019

\begin{abstract}
Functional handwriting is a process involving various complex interactions between physical, cognitive and sensory systems. Since muscular motion is of a peculiar nature for each person, handwriting properties, such as pencil pressure and speed of writing, can be considered as a unique marker of identity. Moreover, impairments of handwriting in many cases are connected to neurodevelopmental disorders such as attention deficit hyperactivity disorder, developmental coordination disorder, autism spectrum disorders, Parkinson's disease, etc. From this point of view, investigations of handwriting kinematics and pressure can be highly important for both forensic science and medicine. Commonly, the kinematic and pressure features of handwriting are evaluated using a graphics tablet with a stylus or electronic pens. The production of such devices is quite expensive. Therefore, the development of new methods for individual handwriting analysis is an important and current goal. Laser speckle contrast imaging (LSCI) is a powerful method, which is sensitive to both motion and pressure. Since the developed technique requires the use of only a simple laser diode and camera for image acquisition, LSCI is a cost-effective and practical tool for handwriting analysis. In the current letter we present a robust LSCI-based method for handwriting pressure and kinematics evaluation. The introduced approach was validated by an Archimedean spiral writing task.
\end{abstract}

Keywords: laser speckle imaging, handwriting, autism, pressure, speckle patterns, light scattering, forensics 


\section{Introduction}

Handwriting is a functional skill used for communication, self-expression and the recording of thoughts, among other things, and it simultaneously involves both motor and cognitive processes [1]. Most people typically learn how to write in childhood, developing individuality and automaticity of handwriting such that the motor processes do not interfere with the cognitive ones. In the literature, it has been shown that handwriting quality is significantly correlated with academic achievement during school years [2-4], self-esteem [3], psychosocial well-being [5], etc.

Moreover, it has been shown that handwriting difficulties can be a sign of neurodevelopmental disorders such as attention deficit hyperactivity disorder [6], developmental coordination disorder [7], autism spectrum disorders [8] etc. Also, the investigation of handwriting peculiarities can be helpful for the early stage diagnostics of Parkinson's disease [9]. All of these diseases are associated with difficulties in the coordination and performance of skilled motor tasks.

The hand motion features of different people can be characterized by their muscular structure and cognitive control characteristics. In this way, handwriting properties, such as speed of writing and pencil pressure, can be considered to be a unique marker of identity. From this point of view, studies of handwriting feature determination are highly useful for personal identification [10]. Therefore, the identification of meaningful parameters of individual handwriting can be very important for both criminology and medicine.

Typically, the kinematic and pressure features of handwriting are evaluated using a graphics tablet with a stylus [11]. However, due to the relatively large thickness of the tablet, handwriting cannot be performed properly, critically affecting the accuracy of any measurements. Thus, the individual's unique handwriting characteristics can be lost. In order to solve this problem, different types of electronic pens have been developed to evaluate kinematic and pressure features [12]. The production of such devices, including graphics tablets and electronic pens, is quite expensive. Therefore, the development of a new method of handwriting analysis is important and extremely current.

Laser speckle contrast imaging (LSCI) is a powerful method mostly used for the non-invasive real-time mapping/diagnosis of flow [13]. LSCI has been successfully implemented for different biomedical applications such as cerebral blood flow monitoring [14, 15], vascular biology $[16,17]$, tumor screening [18], the monitoring of weak allergen reactions [19-21], clinical use on skin [22], and others. In addition, LSCI is effectively used in industry for paint drying monitoring and roughness measurements [23, 24].

Laser speckle is a random phenomenon which has been statistically described by Goodman [25]. The spatio-temporal coherence properties of light scattered by rough surfaces lead to intensity fluctuations. The image formed at a given point of the observation plane can be described as a superposition of the amplitude spread functions. Each of these spread functions arises from a different scattering point on the observed surface. Thus, a highly complex pattern of interference arises,

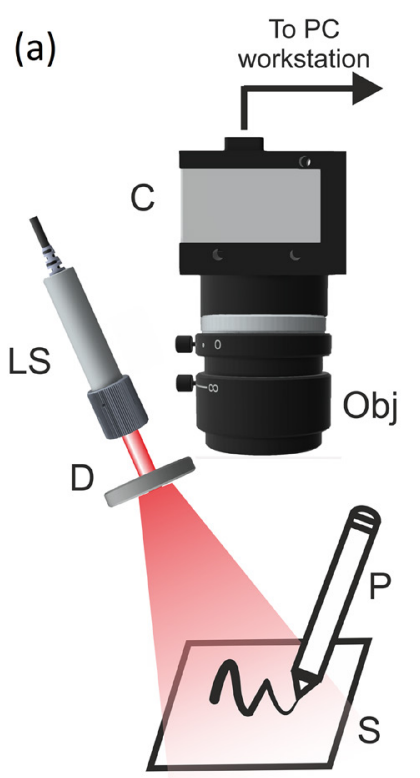

(b)
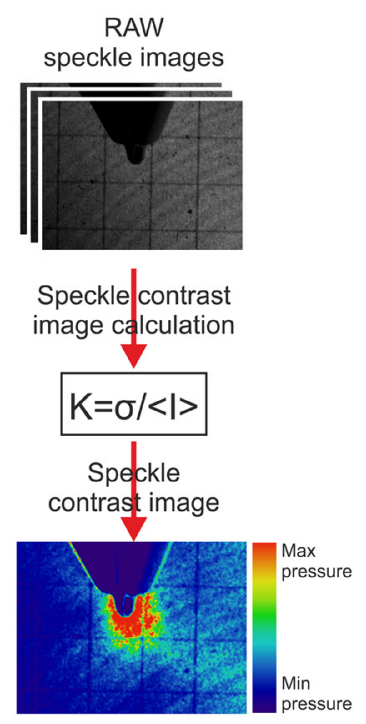

Figure 1. The LSCI system scheme and principles of operation. (a) Schematic presentation of LSCI scheme: C-CCD camera, LS - light source, D—diffuser, Obj—objective, $\mathrm{P}$ - pen, S-sample of paper; (b) the principle of speckle contrast image calculation: CCD-camera-captured stack of raw speckle images. Furthermore, the speckle contrast images calculated using equation (1) by a custom-developed algorithm in the offline regime are shown. The scale bar is equal to $5 \mathrm{~mm}$.

known as a laser speckle pattern. If any parts of the illuminated surface are moving, if the roughness of the surface fluctuates, or if some pressure is applied to the surface, it introduces temporal fluctuations in the single speckle intensity. Captured by CCD or CMOS camera with a finite exposure time, these fluctuations manifest the blurring of a speckle pattern. Such blurring leads to a reduction in speckle contrast, which is calculated using the equation [25]:

$$
K=\frac{\sigma}{\langle I\rangle},
$$

where $K$ is the speckle contrast, $\sigma$ is the intensity standard deviation and $\langle I\rangle$ is the mean intensity.

Speckle contrast imaging can be used (utilizing statistical analysis of the blurring degree) for the evaluation of the motion of scattering centers localized within an object of interest, and, therefore, for the assessment of local pressure applied to the object. The higher speed of scattering center displacement within the object and the external pressure applied to its surface produce a higher blurring degree. Therefore, changes of local pressure to the surface of the observed object produce corresponding changes in the speckle patterns and, respectively, in the speckle contrast.

LSCI is a simple method utilizing a coherent laser source (in particular, a laser diode) for object illumination and CCD or a CMOS camera for raw speckle image acquisition. In fact, it has been shown that even a web camera and smartphone camera can be used for LSCI [26, 27]. In the current paper, we introduce a simple and robust LSCI-based method for handwriting evaluation as an alternative to the available approaches. 


\section{Experimental setup}

The experimental setup, presented in figure 1, has been used for the evaluation of handwriting kinetics and pen pressure.

A 13 mW laser diode (RLD650-13-3, Roithner Lasertechnik $\mathrm{GmbH}$, Austria) emitting light at $655 \mathrm{~nm}$, which was further expanded by a Thorlabs Engineered Diffuser (ED1-C20, Thorlabs, USA), illuminates the sheet of paper (see figure 1). Further, a CMOS camera (DCC3240M, $1280 \times 1024$, pixel size of $6.7 \mu \mathrm{m}$, Thorlabs, USA) used in combination with a $12 \mathrm{~mm}$ F1.4 objective (Kenko Tokina Co., Ltd, Japan) has been used for the acquisition of gray-scale raw speckle images. The obtained images were processed by a custom-developed algorithm in the offline regime using a Fiji/Image J (image processing package) and MATLAB r2018b software environment.

For the acquisition of speckle contrast images two methods are typically applied. The first one, and the most commonly used method, is spatial analysis utilizing a sliding window over the raw speckle image [28]. Sliding windows with a size of $5 \times 5$ and $7 \times 7$ pixels are usually used for the spatial processing of each raw image in a captured image sequence using equation (1). Further, the obtained speckle contrast images are averaged for noise signal reduction. However, using a sliding window leads to a loss of spatial resolution in the final speckle contrast image. To avoid a reduction in spatial resolution, an alternative temporal analysis method has been introduced [29]. To perform temporal processing speckle contrast, the values should be calculated using equation (1) for each pixel of the raw speckle image sequence. Thus, temporal processing allows us to preserve the initial resolution of the final speckle contrast image. In this way, in the current work, the temporal processing has been chosen as optimal.

\section{Results and discussion}

To demonstrate the possibility of evaluating handwriting pressure changes the following experiments have been performed. The laser diode illuminates the sheet of paper. The volunteer periodically presses the paper with the tip of a pen in a similar way to handwriting for $10 \mathrm{~s}$. A CCD camera captures this process over $1 \mathrm{~ms}$ exposure time. Further, the sequence of time-dependent speckle contrast images has been calculated. To calculate each speckle contrast image ten raw speckle frames were used. The obtained speckle contrast images were converted into 8-bit images so that the lower speckle contrast values, and, respectively, higher pressure, correspond to the higher image intensity values. Further, these 8-bit images were colored using a false color palette for better clarity. Blue corresponds to the absence of pressure and red corresponds to maximal pressure.

It is clearly seen from figure 2(a) that pressing a paper sheet with a pen tip leads to the appearance of an area of increased intensity around the tip corresponding to decreased speckle contrast values and, thus, to the presence of pressure. Therefore, the analysis of time-dependent speckle contrast values provides information about the relative changes of local pressure induced by the pen during handwriting.

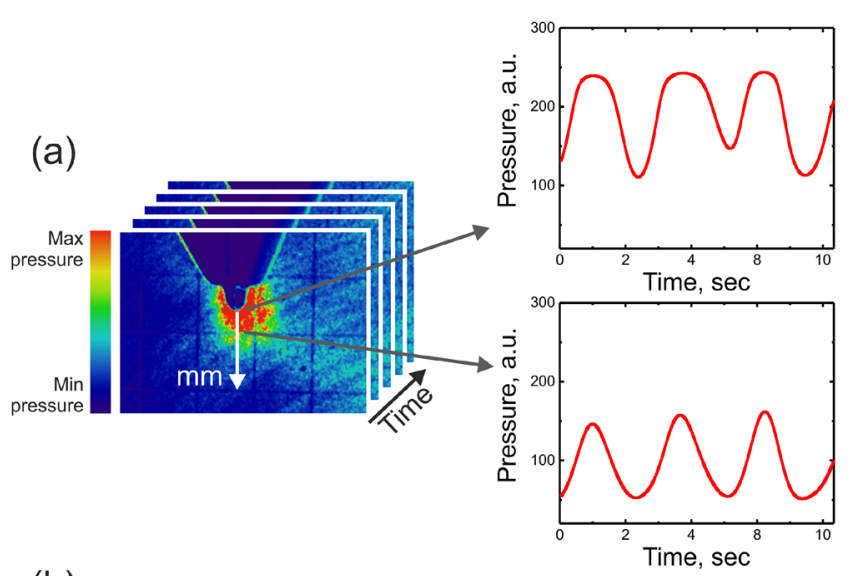

(b)

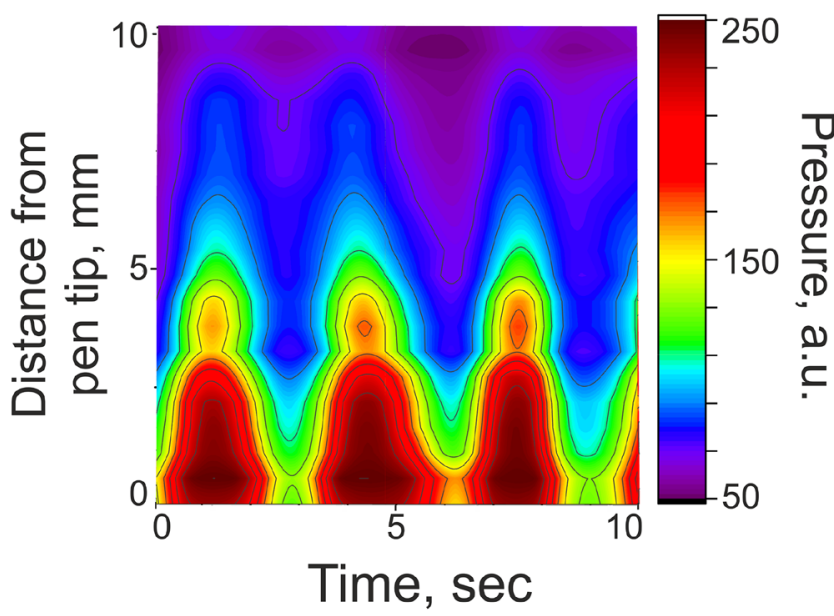

Figure 2. An example of time-dependent pressure change analysis for experiments with the periodical pressing of paper by a pen tip. (a) The speckle contrast image and time- dependent pressure change profiles for points located at distances $1 \mathrm{~mm}$ and $3 \mathrm{~mm}$ from the pen tip. (b) The surface corresponding to the time-dependent pressure changes for points located at distances $0 \mathrm{~mm}-10 \mathrm{~mm}$ from the pen tip.

To obtain such information, the intensity values along the $10 \mathrm{~mm}$ length vector (represented as white arrows in figure 2(a)) directed from the end of the pen tip to the bottom of the frame were calculated for each obtained speckle contrast image. Figure 2(a) shows the time-dependent profiles for the described experiment at the points located at distances $1 \mathrm{~mm}$ and $3 \mathrm{~mm}$ from the pen tip. Figure 2(b) shows the surface plot corresponding to the time-dependent pressure profiles along the $10 \mathrm{~mm}$ length vector directed from the end of the pen tip to the bottom of the frame. It is clearly seen that local pressure induced by the pen decreased from the pen tip to the bottom of the frame. It should be pointed out that the vector for pressure evaluation has been chosen only in the area corresponding to the paper sheet not the pen tip itself. Since movement of the pen influences the speckle contrast only in the localized area corresponding to the pen tip, the pressure evaluation was not interrupted by the movement of the pen. Arguably, the proposed analysis of the observed speckle patterns can be used as a tool for the diagnosis of particular mental diseases and/or to determine relative pressure variations during the handwriting evaluation of an individual. 
(a)

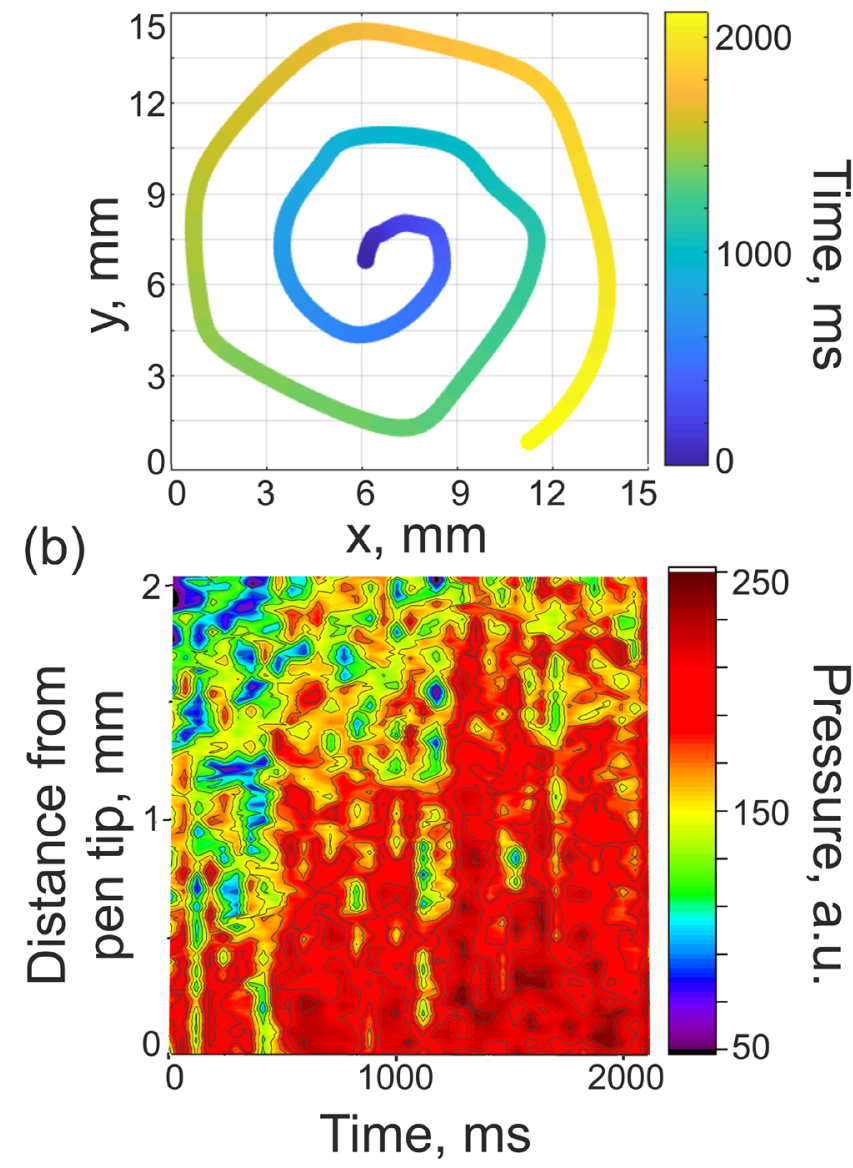

Figure 3. An example of handwriting kinematics and pressure analysis during the Archimedean spiral writing task. (a) The colorcoded time-dependent trajectory of the Archimedean spiral writing task. (b) The surface corresponding to the time-dependent pressure changes for the points located at distances $0 \mathrm{~mm}-2 \mathrm{~mm}$ from the pen tip.

In addition, the following experiment has been performed to demonstrate the possibility of evaluating both handwriting kinematics and pressure changes. In a similar way the laser diode illuminates the sheet of paper. The volunteer drew the Archimedean spiral using a pen. The Archimedean spiral is one of the most popular handwriting exercises used to evaluate motor performance in various movement disorders $[30,31]$. As the writing data are captured by the camera, the trajectory of the pen tip can also be easily monitored utilizing a motion tracking algorithm [32]. Moreover, it is also possible to assess the speed of handwriting performance. Figure 3(a) shows the trajectory of the pen tip during the performance of a handwriting task. The trajectory has been tracked using an MTrackJ plugin for ImageJ software. The trajectory line has been color-coded in a way so that the changing color corresponds to a time value from the beginning of the handwriting task. It is clearly seen that the inner spiral turn takes more time to be performed than the outer one. Thus, the speed of writing is different for the various parts of the performed figure.
To estimate the pressure pattern during the Archimedean spiral exercise the same method described above has been applied. The surface plot corresponding to the time- dependent pressure profiles has been plotted for a $2 \mathrm{~mm}$ length vector directed from the pen tip to the bottom of the frame (see figure 3(b)). As well as in the previous experiment, the vector was only plotted in the area corresponding to the paper sheet not the pen tip itself. So, all data were collected from the static area where the speckle contrast was influenced only by the changes in pressure but not by the motion of the pen. It is clearly seen that the local pressure caused by the pen tip changes during the writing task.

The analysis of kinematic and pressure patterns obtained by the proposed LSCI method allows us to distinguish the handwriting performed by different people and also allows us to diagnose the early stage of neurodevelopmental disorders.

Thus, we introduce the concept of evaluating an individual's handwriting with the LSCI approach. To become a practical tool for routine day-to-day use, the proposed method requires further development. Particularly, calibration of the speckle contrast values on the real pressure caused by the pen on the surface of the paper should be performed. It should also be pointed out that the proposed approach can be used in a back-reflected mode, with the illumination and detection of the scattered light performed from the underside of a transparent glass table and a paper sheet placed on the surface.

\section{Conclusion}

We present a simple and robust experimental method for the evaluation of handwriting pressure and kinematics by using LSCI. Since the experimental implementation of the introduced technique requires the use of only a simple laser diode and standard camera for image acquisition, the method is a cost effective, affordable and practical tool for the express assessment of handwriting features. The proposed technique was verified by an Archimedean spiral writing task. Thus, the LSCI, to the best to our knowledge, has been used for the first time to examine handwriting features. The proposed technique still requires further development to become a practical tool for routine day-to-day handwriting assessment. We also believe that LSCI-based handwriting evaluation has great potential and will find an application in the diagnosis of neurodevelopmental disorders.

\section{Acknowledgments}

The research of Vyacheslav Kalchenko was supported by The Henry Chanoch Krenter Institute for Biomedical Imaging and Genomics (Staff Scientists grant program). The authors acknowledge partial support from INFOTECH and the Academy of Finland (grant projects: 326204), the MEPhI Academic Excellence Project (contract no. 02.a03.21.0005), and the National Research Tomsk State 170 University Academic DI Mendeleev Fund Program. Anton Sdobnov was supported by a Finnish Cultural Foundation (00180998) grant. 


\section{References}

[1] Jones D and Christensen C 1999 Relationship between automaticity in handwriting and students' ability to generate written text J. Educ. Psychol. 91 44-9

[2] Cahill S 2009 Where does handwriting fit in? Strategies to support academic achievement Intervent. Sch. Clin. 44 223-9

[3] Feder K and Majnemer A 2007 Handwriting development, competency, and intervention Dev. Med. Child Neurol. $49312-7$

[4] Graham S and Weintraub N 1996 A review of handwriting research: progress and prospects from 1980 to 1994 Educ. Psychol. Rev. 8 7-87

[5] Cornhill H and Case-Smith J 1996 Factors that relate to good and poor handwriting Am. J. Occup. Ther. 50 732-9

[6] Racine M, Majnemer A, Shevell M and Snider L 2008 Handwriting performance in children with attention deficit hyperactivity disorder (ADHD) J. Child Neurol. 23 399-406

[7] Rosenblum S, Epsztein L and Josman N 2008 Handwriting performance of children with attention deficit hyperactive disorders: a pilot study Phys. Occup. Ther. Pediatr. 28 219-34

[8] Fuentes C, Mostofsky S and Bastian A 2009 Children with autism show specific handwriting impairments Neurology 73 1532-7

[9] Drotár P, Mekyska J, Rektorová I, Masarová L, Smékal Z and Faundez-Zanuy M 2016 Evaluation of handwriting kinematics and pressure for differential diagnosis of Parkinson's disease Artif. Intell. Med. 67 39-46

[10] Martens R and Claesen L 1997 On-line signature verification: discrimination emphasised Proc. 4th Int. Conf. on Document Analysis and Recognition (August 1997) vol 2 pp $657-60$

[11] Yamazaki Y, Mizutani Y and Komatsu N 1999 Extraction of personal features from stroke shape, writing pressure and pen inclination in ordinary characters Proc. of the 5th Int. Con. on Document Analysis and Recognition (September 1999) vol PR00318 pp 426-9

[12] Kikuchi M and Akamatsu N 2001 Development of speedy and high-sensitivity pen system for writing pressure and writer identification Syst. Comput. Japan 32 1-12

[13] Sdobnov A, Bykov A, Molodij G, Kalchenko V, Jarvinen T, Popov A and Meglinski I 2018 Speckle dynamics under ergodicity breaking J. Phys. D: Appl. Phys. 51155401

[14] Kalchenko V, Israeli D, Kuznetsov Y and Harmelin A 2014 Transcranial optical vascular imaging (TOVI) of cortical hemodynamics in mouse brain Sci. Rep. 45839

[15] Kalchenko V, Sdobnov A, Meglinski I, Kuznetsov Y, Molodij G and Harmelin A 2019 A robust method for adjustment of laser speckle contrast imaging during transcranial mouse brain visualization Photonics 680

[16] Kalchenko V, Ziv K, Addadi Y, Madar N, Meglinski I, Neeman M and Harmelin A 2010 Combined application of dynamic light scattering imaging and fluorescence intravital microscopy in vascular biology Laser Phys. Lett. 7 603-6
[17] Kalchenko V, Kuznetsov Y, Meglinski I and Harmelin A 2012 Label free in vivo laser speckle imaging of blood and lymph vessels J. Biomed. Opt. 17050502

[18] Kalchenko V, Madar N, Meglinski I and Harmelin A 2011 In vivo characterization of tumour and tumour vascular network using a multi-mode imaging approach J. Biophoton. 4 645-9

[19] Kalchenko V, Kuznetsov Y, Prise D, Meglinski I and Harmelin A 2014 Mouse ear swelling test for weak allergens by using laser speckle imaging with the long exposure time J. Biomed. Opt. 1960502

[20] Kuznetsov Y, Kalchenko V, Astafeva N and Meglinski I 2014 Optical diagnostics of vascular reactions triggered by weak allergens using the laser speckle imaging contrast technique Quantum Electron. 44 713-8

[21] Kalchenko V, Meglinski I, Sdobnov A, Kuznetsov Y and Harmelin A 2019 Combined laser speckle imaging and fluorescent intravital microscopy for monitoring acute vascular permeability reaction J. Biomed. Opt. 24060501

[22] Roustit M, Millet C, Blaise S, Dufournet B and Cracowski J 2010 Excellent reproducibility of laser speckle contrast imaging to assess skin microvascular reactivity Microvasc. Res. 80 505-11

[23] Leonard L and Toal V 1998 Roughness measurement of metallic surfaces based on the laser speckle contrast method Opt. Lasers Eng. 30 433-40

[24] Arizaga R, Grumel E, Cap N, Trivi M, Amalvy J, Yepes B and Ricaurte G 2006 Following the drying of spray paints using space and time contrast of dynamic speckle J. Coat. Technol. Res. 3 295-9

[25] Goodman W 1975 Statistical properties of laser speckle patterns Laser Speckle and Related Phenomena (Berlin: Springer)

[26] Willenz U, Ragol S, Remer I, Shoham Y, Hazan S, Sinelnikov I and Bilenca A 2015 Static laser speckle contrast analysis for noninvasive burn diagnosis using a camera-phone imager J. Biomed. Opt. 20086009

[27] Richards L, Kazmi S, Davis J, Olin K and Dunn A 2013 Low-cost laser speckle contrast imaging of blood flow using a webcam Biomed. Opt. Express 4 2269-83

[28] Zakharov P, Scheffold F and Weber B 2014 Laser speckle imaging of cerebral blood flow Optical Imaging of Neocortical Dynamics vol 85, ed B Weber and F Helmchen (Totowa, NJ: Humana Press) pp 255-71

[29] Cheng H, Luo Q, Zeng S, Chen S, Cen J and Gong H 2003 Modified laser speckle imaging method with improved spatial resolution J. Biomed. Opt. 8 559-64

[30] Saunders-Pullman R, Derby C, Stanley K, Floyd A, Bressman S and Lipton R 2008 Validity of spiral analysis in early Parkinson's disease Mov. Disorders 23 531-7

[31] Stanley K, Hagenah J, Bruggemann N, Reetz K, Severt L and Klein C 2010 Digitized spiral analysis is a promising early motor marker for Parkinson's disease Parkinsonism Relat. Disorders 16 233-4

[32] Murray D and Basu A 1994 Motion tracking with an active camera IEEE Trans. Pattern Anal. Mach. Intell. 16 449-59 